\title{
SHAPELETS: A NEW METHOD TO MEASURE GALAXY SHAPES $^{a}$
}

\author{
A. Refregier \\ Institute of Astronomy, Madingley Road, Cambridge CBз OHA, UK; \\ ar@ast.cam.ac.uk \\ T.-C. Chang \\ Dept. of Astronomy, 550 W. 120 street, Columbia University, New York, \\ NY 10027, USA; tchang@astro.columbia.edu \\ D. J. Bacon ${ }^{b}$ \\ Institute for Astronomy, University of Edinburgh, Blackford Hill, \\ Edinburgh EH9 3HJ, UK; djb@roe.ac.uk
}

\begin{abstract}
We present a new approach to measure the shapes of galaxies, a fundamental task in observational astronomy. This approach is based on the decomposition of a galaxy image into a series of orthogonal basis functions, or 'shapelets'. Our choice of basis functions, namely the Gauss-Hermite series, has a number of remarkable properties under distortions, convolutions and noise, which makes them particularly well suited for astrophysical applications. In particular, we describe how they can be used to measure the shear induced by weak gravitational lensing, with the precision required for upcoming surveys. We also show how shapelets can be used to reconstruct images from interferometric observations. Other application of shapelets, such as image compression, PSF deconvolution, de-projection and the study of galaxy morphology, are also briefly discussed.
\end{abstract}

\section{Introduction}

The measurement of galaxy shapes is a fundamental task in observational astronomy. In these proceedings, we present a new method for shape measurements described in detail in Refregier (2001). It is based on the linear decomposition of each galaxy into a series of localised basis functions with different shapes, which we call 'Shapelets'. As a basis set, we choose the GaussHermite series, whose remarkable properties make it ideal for astronomical applications. In particular, we summarise the results of Refregier \& Bacon (2001) who showed how shapelets can be used to measure weak lensing, with the precision required for upcoming surveys. We also describe how shapelets can be used to reconstruct images from interferometric data, as described by Chang \& Refregier (2001).

${ }^{a}$ To appear in Procs. of the Workshop "The Shapes of Galaxies and their Halos", Yale, May 2001.

${ }^{b}$ previous address: Institute of Astronomy, Madingley Road, Cambridge CB3 OHA, UK. 
We illustrate the method using images from the Hubble Space Telescope and from the FIRST radio survey. Finally, we describe several further applications of shapelets and discuss their relevance in the context of astronomical applications requiring high precision.

\section{Shapelet Method and Properties}

We begin by summarising the formalism of Refregier (2001) for a description of galaxies in the Gauss-Hermite basis set. A galaxy with intensity $f(\mathbf{x})$ can be decomposed into our basis functions $B_{\mathbf{n}}(\mathbf{x} ; \beta)$ as

$$
f(\mathbf{x})=\sum_{\mathbf{n}} f_{\mathbf{n}} B_{\mathbf{n}}(\mathbf{x} ; \beta),
$$

where $\mathbf{x}=\left(x_{1}, x_{2}\right)$ and $\mathbf{n}=\left(n_{1}, n_{2}\right)$. The 2-dimensional cartesian basis functions can be written as $B_{\mathbf{n}}(\mathbf{x} ; \beta)=B_{n_{1}}\left(x_{1} ; \beta\right) B_{n_{2}}\left(x_{2} ; \beta\right)$, in terms of the 1dimensional basis functions

$$
B_{n}(x ; \beta) \equiv\left[2^{n} \pi^{\frac{1}{2}} n ! \beta\right]^{-\frac{1}{2}} H_{n}\left(\frac{x}{\beta}\right) e^{-\frac{x^{2}}{2 \beta^{2}}},
$$

where $H_{n}(x)$ is a Hermite polynomial of order $n$. The parameter $\beta$ is a characteristic scale, which is typically chosen to be close to the radius of the object. These basis functions are the eigenstates of the Quantum Harmonic Oscillator (QHO), allowing us to use the formalism developed for this problem. Similar decomposition into basis functions has been independently suggested by Bernstein \& Jarvis (2001). The first few basis functions are shown in Figure 1 .

Because these basis functions, or 'shapelets', form a complete orthonormal set, the coefficients $f_{\mathbf{n}}$ can be found using

$$
f_{\mathbf{n}}=\int_{-\infty}^{\infty} d^{2} x f(\mathbf{x}) B_{\mathbf{n}}(\mathbf{x} ; \beta)
$$

Figure 2 shows an example of the decomposition of a galaxy in the Hubble Deep Field (Williams et al 1996). The details of the image are fully reconstructed if coefficients up to $n=20$ are included; this decomposition thus provides an excellent and efficient description of galaxy images in practice.

The chosen basis functions have a number of remarkable properties. Firstly, they are invariant under Fourier transforms up to a scaling factor, so that

$$
\widetilde{B}_{n}(k ; \beta)=i^{n} B_{n}\left(k ; \beta^{-1}\right),
$$

where tildes denote Fourier transforms. As a result, convolutions (which correspond to products in Fourier space) can be simply expressed using shapelets. 


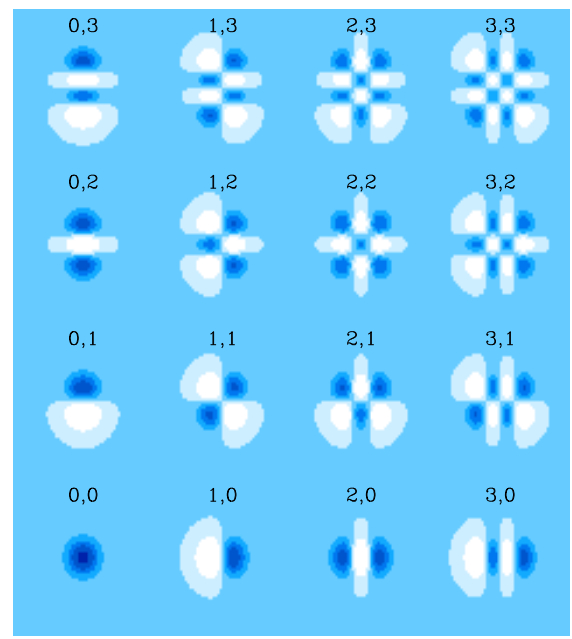

Figure 1: First few 2-dimensional Cartesian basis functions $B_{n_{1}, n_{2}}$. The dark and light regions correspond to positive and negative values, respectively.

Let us consider the convolution $h(x)=(f * g)(x)$ of 2 functions. Each function can be decomposed into shapelet coefficients with scales $\alpha, \beta$ and $\gamma$, which are then related by

$$
h_{n}=\sum_{m, l=0}^{\infty} C_{n m l} f_{m} g_{l},
$$

where the convolution tensor $C_{n m l}(\alpha, \beta, \gamma)$ can be estimated analytically using a simple recursion relation (Refregier \& Bacon 2001).

Shapelets also have simple properties under coordinate transformations such as translation, dilation, rotation and shear. For instance, let us consider the distortion of an image $f(\mathbf{x})$ under the action of a weak shear $\gamma_{i}$, as occurs in weak gravitational lensing. To first order in the shear, the distorted image can be written as

$$
f^{\prime} \simeq\left(1+\gamma_{i} \hat{S}_{i}\right) f
$$

where $\hat{S}_{i}$ is the shear operator. It is easy to show that this operator takes a simple form in shapelet space, namely

$$
\hat{S}_{1}=\frac{1}{2}\left(\hat{a}_{1}^{\dagger 2}-\hat{a}_{2}^{\dagger 2}-\hat{a}_{1}^{2}+\hat{a}_{2}^{2}\right), \quad \hat{S}_{2}=\hat{a}_{1}^{\dagger} \hat{a}_{2}^{\dagger}-\hat{a}_{1} \hat{a}_{2},
$$

where $\hat{a}_{i}^{\dagger}$ and $\hat{a}_{i}$ are the raising and lowering operators in the QHO formalism, 


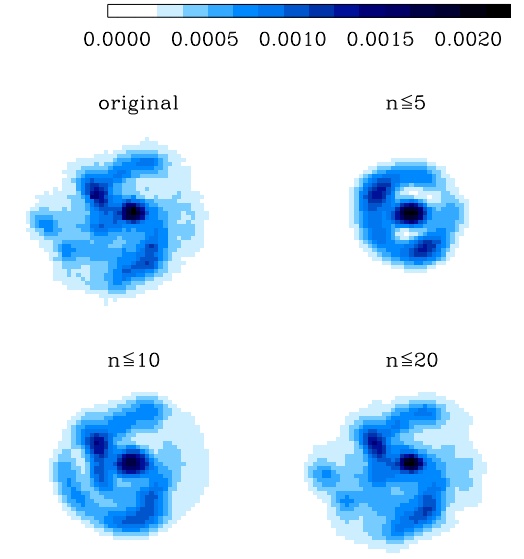

Figure 2: Decomposition of a galaxy image found in the HDF. The original $60 \times 60$ pixel HST image (upper left-hand panel) can be compared with the reconstructed images with different maximum order $n=n_{1}+n_{2}$. The shapelet scale is chosen to be $\beta=4$ pixels. The lower right-hand panel $(n \leq 20)$ is virtually indistinguishable from the initial image.

for each dimension $i=1,2$. Similar operators can be constructed for the other coordinate transformations.

\section{Measurement of Weak Lensing}

The weak distortions induced by lensing on the images of background galaxies provide a direct measure of the distribution of mass in the Universe. This weak lensing method is now routinely used to study galaxy clusters, and has recently been detected in the field (Wittman et al 2000; Bacon et al 2000; Kaiser et al 2000; Maoli et al 2001; van Waerbeke et al 2000, 2001; Rhodes et al. 2001). Because the lensing effect is only of a few percent on large scales, a precise method for measuring the shear is required. The original methods of Bonnet \& Mellier (1995) and Kaiser, Squires \& Broadhurst (KSB; 1995) are not sufficiently accurate and stable for the upcoming weak lensing surveys (see Bacon et al 2001, Erben et al 2001). Thus several new methods have been proposed (Kuijken 1999; Rhodes, Refregier \& Groth 2000; Kaiser 2000; Bernstein \& Jarvis 2001).

The remarkable properties of our basis functions make shapelets particularly well suited for providing the basis of a new method for measuring the 
shear (Refregier \& Bacon 2001). First, the Point-Spread Function (PSF) of the instrument can be modeled by decomposing stellar images into shapelets and by interpolating the resulting coefficients across an image. Galaxy images can then themselves be decomposed into shapelet coefficients, and the analytical form of the convolution matrix (see Eq. [1) can be used to deconvolve the Point-Spread Function. From the properties of shapelets under shears (eq. [7]), one can then construct a linear estimator for the shear from the (deconvolved) coefficients of the galaxies of the form

$$
\widetilde{\gamma}_{i \mathbf{n}}=\frac{f_{\mathbf{n}}^{\prime}-\left\langle f_{\mathbf{n}}\right\rangle}{S_{i \mathbf{n m}}\left\langle f_{\mathbf{m}}\right\rangle}
$$

where $f_{\mathbf{n}}^{\prime}$ are the lensed coefficients, the brackets denote an average over the unlensed galaxy population, and $\mathbf{n}$ is even (odd) for the $\gamma_{1}\left(\gamma_{2}\right)$ component of the shear. The tensor $S_{i \mathbf{n m}}$ is the matrix representation of the shear operators of Equation (7). It is easy to show that these estimators are unbiased, i.e. that $\left\langle\widetilde{\gamma}_{\mathbf{i n}}\right\rangle=\gamma_{i}$, if the unlensed galaxies are randomly oriented. These estimators can then be combined to construct a minimum variance global estimator for the shear over a sample of galaxies.

To test the method, we used the image simulations described in Bacon et al. (2001). These were designed to reproduce the observational conditions of ground based telescopes such as appropriate throughput, PSF and noise, along with the statistics of the galaxy population observed in HST images. An artificial shear was applied to the simulated galaxies, and the shapelet method was used on the resulting realistic images to recover the applied shear. The recovered shear values from the simulations are plotted as a function of the output shear in Figure 3. Clearly, the method is unbiased, and is found to be robust with PSF shape and size. It thus provides an improvement over the KSB method which was shown to have small but significant instabilities and biases (Bacon et al 2001; Erben et al. 2001).

\section{Image reconstruction with Interferometers}

Another application of shapelets is the reconstruction of images from interferometric data, as described in detail in Chang \& Refregier (2001). Interferometric data are collected in the $u v$ space, the Fourier-transform of the sky surface brightness. The observed quantity is the visibility measured for each antenna pair $(i, j)$ at time $t$ and at frequency $\nu$ and is given by

$$
V_{i j}(\nu, t)=\int d^{2} l \frac{A(\mathbf{l}, \nu) f(\mathbf{l}, \nu, t)}{\sqrt{1-|l|^{2}}} e^{-2 \pi i\left[u l+v m+w\left(\sqrt{1-|l|^{2}}-1\right)\right]},
$$




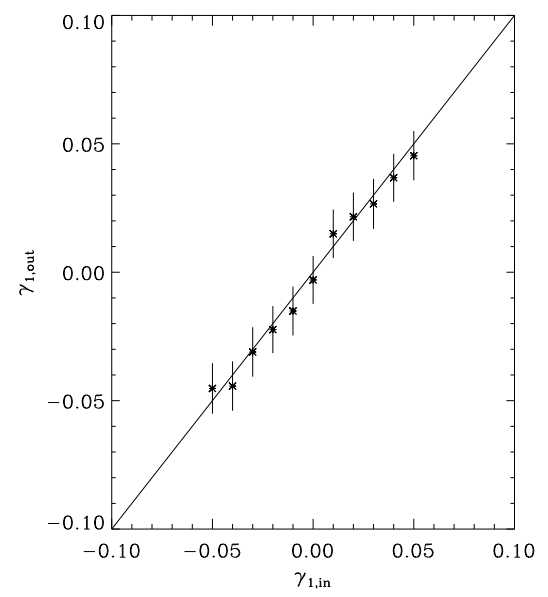

Figure 3: Input shear vs recovered shear for a set of 11 simulations. Note the linear relationship between input and recovered shear; the slope is found to be $0.97 \pm 0.04$ for $\gamma_{1}$ and $1.00 \pm 0.04$ for $\gamma_{2}$.

where $f(\mathbf{l}, \nu, t)$ is the surface brightness of the sky at position $\mathbf{l}=(l, m)$ with respect to the phase center, and $A(\mathbf{l}, \nu)$ is the (frequency-dependent) primary beam.

In analogy with the shapelet decomposition in real space, we wish to decompose sources directly in the $u v$ space. However, the discrete and finite sampling of the $u v$ plane prevents a direct linear decomposition of the visibilities $V_{i j}$ into shapelet coefficients. Instead, we simultaneously fit for the shapelet coefficients of a collection of sources on the $u v$ plane, by using a $\chi^{2}$ fit:

$$
\chi^{2}=(\mathbf{d}-\mathbf{M} \mathbf{f})^{T} \mathbf{C}^{-1}(\mathbf{d}-\mathbf{M} \mathbf{f}),
$$

where $\mathbf{d}=\left\{\bar{V}_{i j}\right\}$ is the visibility data vector, $\mathbf{M}=\left\{\bar{V}_{i j}^{\mathbf{n} s}\right\}$ is the theory matrix composed of visibilities corresponding to each basis function, and $\mathbf{f}=\left\{f_{\mathbf{n} s}\right\}$ is the shapelet coefficient vector. The indices $s$ and $n$ stand for the source number and shapelet state, respectively. The covariance error matrix of the visibilities

$$
\mathbf{C}=\operatorname{cov}[\mathbf{d}, \mathbf{d}]=\left\langle(\mathbf{d}-\langle\mathbf{d}\rangle)^{T}(\mathbf{d}-\langle\mathbf{d}\rangle)\right\rangle
$$

is provided by the interferometric hardware. The resulting $\chi^{2}$-fit is linear in its parameters and can therefore be performed by simple matrix operations. The complex effects of bandwidth smearing, time averaging and non-coplanarity of 


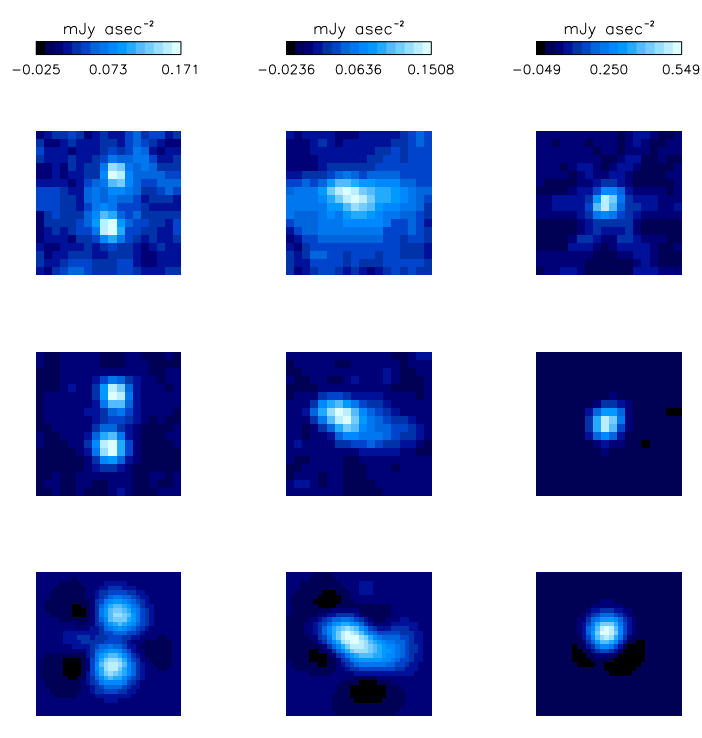

Figure 4: Reconstruction of three sources from one of the FIRST grid pointings. The 'dirty' images (which are direct inverse Fourier transformation of the data), CLEAN images, and shapelet reconstructions are shown from top to bottom, respectively. The images are $32^{\prime \prime}$ across and the resolution is about $5^{\prime \prime} .4$ (FWHM). The dirty and CLEAN images are displayed with a $1^{\prime \prime} .8$ pixel size, while the shapelet reconstruction images have $1^{\prime \prime}$ pixels. For the shapelet reconstruction, we used Wiener filtering and smoothing by a Gaussian restoring beam with a standard deviation of $2.3^{\prime \prime}$.

the array can be fully corrected for in the process.

As an example we consider the observing conditions of the FIRST radio survey (Becker et al. 1995; White et al. 1997). Using one of the FIRST pointings, as shown in Fig. 1, we find that our method compares well with CLEAN, the commonly used method for interferometric imaging. Our method has the advantage of being linear in the shapelet parameters, of fitting all sources simultaneously, and of providing the full covariance matrix of the coefficients, which allows us to quantify the errors and cross-talk in image shapes. It is well-suited for quantitative shape measurements which require high-precision. In particular, combining with the results from the previous section (Refregier \& Bacon 2001), our results provide an accurate method for measuring weak lensing with interferometers. 


\section{Conclusions}

Shapelets provide a new method to measure the shape of galaxies. The chosen basis functions have convenient properties under convolutions and distortions. This makes shapelets ideally suited for the measurement of weak lensing shear, after correcting for the smearing effect of the point spread function. They can also be used to reconstruct images from interferometric data. The method is very general and is ideally suited for astrophysical applications requiring high

precision. In particular, it can be used for image compression, modeling and deconvolution of the point spread function, and de-projection from 2 dimensional to 3 dimensional data. Another immediate application to explore is that of the measurement and classification galaxy morphology. Shapelets could thus help in unifying the different observational and theoretical studies of galaxy shapes presented in this volume.

\section{Acknowledgments}

We thank Richard Ellis, David Helfand and Richard Massey for useful discussions and on-going collaboration. AR was supported by a fellowship from the EEC TMR network on gravitational lensing and by a Wolfson College Fellowship. TC was supported by NSF grant AST-98-0273. DJB was supported at the IfA by a PPARC postdoctoral fellowship.

\section{References}

1. Bacon D., Refregier A., Ellis R., 2000, MNRAS, 318, 625.

2. Bacon D., Refregier A., Clowe D., Ellis R., 2001, MNRAS, 325, 1065.

3. Becker, R.H., White, R.L., Helfand, D.J. 1995, ApJ, 450, 559

4. Bernstein G. M. \& Jarvis M., 2001, accepted by AJ, astro-ph/0107431.

5. Bonnet H. \& Mellier Y., 1995, A\&A, 303, 331.

6. Chang T. \& Refregier A., 2001, submitted to ApJ, astro-ph/0107085.

7. Erben T., van Waerbeke L., Bertin E., Mellier Y., Schnedier P., 2001, A\&A, 366, 717 .

8. Kaiser N., Squires G., Broadhurst T., 1995, (KSB) ApJ, 449, 460.

9. Kaiser N., 2000, ApJ, 537, 555.

10. Kaiser N., Wilson G., Luppino G. A., 2000, submitted to ApJL, preprint astro-ph/0003338.

11. Kuijken K., 1999, A\&A, 352, 355.

12. Maoli R. et al, 2001, A\&A, 368, 766.

13. Refregier A., 2001, submitted to MNRAS, astro-ph/0105178,

14. Refregier A. \& Bacon D., 2001, submitted to MNRAS, astro-ph/0105179. 
15. Rhodes J., Refregier A., Groth E. 2000, ApJ, 536, 79.

16. Rhodes J., Refregier A., Groth E. 2001, to appear in ApJL, preprint astro-ph/0101213

17. van Waerbeke L. et al, 2000, A\&A, 358, 30.

18. Van Waerbeke L. et al, 2001, A\&A, 374, 757.

19. White, R.L., Becker, R.H., Helfand, D.J., Gregg, M.D. 1997, ApJ, 475, 479

20. Williams R. E. et al., 1996, AJ, 112, 1335.

21. Wittman D., Tyson J. A., Kirkman D., Dell'Antonio I., Bernstein G., 2000, Nature, 405, 143. 